http://jmscr.igmpublication.org/home/ ISSN (e)-2347-176x ISSN (p) 2455-0450

crossref DOI: https://dx.doi.org/10.18535/jmscr/v9i1.38

\title{
A Clinical Study on Changes in Iop after Dexamethasone Usage Following Manual Small Incision Cataract Surgery
}

\author{
Authors \\ Dr Divya Prasad*, Dr Lokesh H M \\ Sri Siddhartha Medical College Hospital and Research Centre, Agalakote, Tumkur \\ *Corresponding Author \\ Dr Divya Prasad \\ Junior Resident, Department of Ophthalmology, SSMCH \& RC, Tumkur
}

\begin{abstract}
Introduction: Cataract is any opacification in lens or its capsule. On the basis of cost effectiveness and feasibility for almost all types of cataract, manual small incision cataract surgery (MSICS) is popular. A dreaded complication as post operative endophthalmitis prompts the need for post operative antibiotics and steroids to control infection and prevent inflammation. But steroid induced glaucoma, corticosteroid induced ocular hypertension are the known leading drawbacks of steroid usage. This study aims at detecting the rise in IOP after the usage of Dexamethasone following MSICS.

Study Design: This is a prospective observational study conducted from November 2018 to April 2020. Total of 59 consecutive non glaucomatous senile and presenile, mature and immature cataract were operated by MSICS and started on Dexamethasone eye drops postoperatively for 6 weeks. Evaluation of changes in IOP preoperatively in comparison to the IOP 6 weeks postoperatively, was recorded by Goldmann Applanation Tonometry.

Results: The mean preoperative IOP among the study subjects was $17.05 \mathrm{mmhg}$, which increased to a mean IOP of 19.32mmhg after 6 weeks of dexamethasone usage following small incision cataract surgery.

Conclusion: Dexamethasone is a safe and potent corticosteroid which effectively reduces the post operative inflammation and pain. Dexamethasone is a safe drug which carries very low risk of IOP rise if the schedule is followed. The patient compliance with respect to the frequency of instillation of Dexamethasone is most important to obtain good outcome.

Keywords: Manual small incision cataract surgery, Intraocular Pressure, Dexamethasone.
\end{abstract}

\section{Introduction}

Cataract and glaucoma are the leading causes of blindness in the world accounting to $51 \%$ and $8 \%$ respectively. ${ }^{1}$ The most commonly performed surgical procedures for cataract are manual small incision cataract surgery (MSICS) and phacoemulsification.
Accordingly, MSICS has gained popularity due to its cost effectiveness, feasibility, wider applicability, shorter learning curve and early rehabilitation. But MSICS has its own disadvantages, of which changes in intraocular pressure is an important one. One among the known postoperative complications is the transient rise in IOP in the immediate post operative period 
which occurs due to the blockage of trabecular meshwork by the retained viscoelastics or by the retained lenticular matter.

Cataract surgery in the present times offers faster wound healing with less postoperative complications and rapid visual recovery time with the help of corticosteroids which are used after cataract surgery. Corticosteroids, by interfering with phospholipase A2 are able to inhibit the arachidonic acid cascade preventing the inflammatory response and reducing the postoperative pain. Corticosteroids also help in preventing the most serious and potentially sight threatening consequence of uncontrolled inflammation, which is cystoid macular edema. But a matter of concern is the rise in IOP which occurs as an adverse event following steroid usage. This adverse event is most commonly seen among the steroid responsive individuals. Persistent elevation of IOP can damage the optic nerve and produce visual field defects, resulting in glaucoma. Factors which play a role in the elevation of IOP are the compliance of the patient, frequency of instillation of the drug, steroid responsiveness of the individual. To prevent these untoward complications, the study of changes in intraocular pressure following steroid usage is of dire importance and is the least explored aspect. Hence, the present study aimed to study the IOP changes occurred following the steroid usage for 6 weeks after MSICS in comparison to the preoperative IOP.

\section{Materials and Methods}

An approximately of 59 cases of cataract as specified under inclusion and exclusion criteria attending Ophthalmology OPD were enrolled in the study.

\section{Inclusion Criteria}

Patients undergoing small incision cataract surgery for senile cataract and presenile cataract mature and immature type

\section{Exclusion Criteria}

Following patients will be excluded from the study:-
- Congenital cataract

- Complicated cataract

- Patients with glaucoma

- Previous anterior chamber abnormality or angle surgery

- Exfoliation and pseudoexfoliation

- Past history of ocular trauma

- Any intraoperative complication

Patient evaluation:

Detailed history of presenting ocular complaints, history of any systemic disorder, recording of their visual acuity by Snellen's vision chart for distant vision and Snellen's near vision chart for near vision.

Slit lamp examination: examination of adnexa, cornea, conjunctiva was done. Size, shape and reactivity of pupil and any disorders like pseudoexfoliation were assessed. Examination of the anterior chamber and the type of cataract with nuclear sclerosis grading based on Lens Opacities Classification System III was done, intraocular pressure was measured by Goldmann Applanation Tonometry.

PCIOL power calculation and biometric reading done by keratometry and Amplitude Scan( Ascan). PCIOL power was calculated using SRK II formula.

Procedure followed for small incision cataract surgery:

The operative procedures were done in the morning hours between 9 am to $12 \mathrm{pm}$.

Peribulbar block with $2 \%$ lignocaine with adrenaline( 1 in 1 lac units) mixed with hyaluronidase $75 \mathrm{IU} / \mathrm{ml}$ and $0.5 \%$ bupivacaine in a ratio of 2:1 was given. Digital ocular massage was given for $10 \mathrm{~min}$.

Operative eye was painted and draped, universal eye speculum inserted, superior rectus bridle suture taken, A fornix based conjunctival flap from 10 to 1 'o clock position was made, hemostasis achieved by ball point cautery. A sclera superior limbal, frown shaped incision of $6 \mathrm{~mm}-7 \mathrm{~mm}$, approximately $1.5 \mathrm{~mm}$ away from the limbus was made. Sclero-corneal tunnel made with a $2.6 \mathrm{~mm}$ crescent. Side port entry made at 
9'o clock hours. Dispersive sterile viscoelastic devices (2\% HPMC) was used as and when required by the surgeon. Continuous curvilinear capsulorrhexis or envelop capsulotomy was done with a cystitome through the side port depending on the maturity of cataract. Entry made into the anterior chamber using a $2.8 \mathrm{~mm}$ entry keratome, followed by adequate hydrodissection with BSS, rotation of the nucleus and prolapse of the nucleus in the anterior chamber, nucleus delivery by wire vectis and sinskey hook (sandwich method), cortical wash given with Simcoe's cannula, $6 \mathrm{~mm}$ all PMMA posterior chamber IOL inserted into the capsular bag. Dialing of the PCIOL was done. Anterior chamber wash given, Side port hydration done and subconjunctival Dexamethasone + Gentamycin injection of $0.3 \mathrm{cc}$ given. Pad and pressure bandage was applied to the eye.

Postoperatively, all the patients developing immediate postoperative complications were excluded from the study. Topical therapy in the form of Dexamethasone and Chloramphenicol eye drops was given in a tapering dose of 10 times a day for 1 week, 8 times a day in the second week, 6 times a day in the third week, 4 times a day in the fourth week, 2 times a day in the fifth week, once a day in the last sixth week. Dark glasses were adviced to be worn by the patients when outdoor. Patients were asked to come for follow up at 1 week and 6 weeks post operation. Measurement of IOP was done at the end of 6 weeks by sterilized Goldmann Applanation Tonometry. All data was collected in preformed proforma.

\section{Results}

In our study Patients ageing less than 60 years were 45 , accounting to $76.30 \%$ constituting the majority of the study population. Patients ageing between 61 to 65 years were 10, accounting to $16.90 \%$ and those more than 65 years were 4 , accounting to $6.80 \%$ of the study population (Table 1) (Graph 1). The mean preoperative IOP among the study subjects was $17.05 \mathrm{mmhg}$, which increased to a mean IOP of $19.32 \mathrm{mmhg}$ after 6 weeks of dexamethasone usage following small incision cataract surgery (Table 2)(Graph 2). The comparison between the pre op IOP with the IOP at 6 weeks following Dexamethasone usage showed that there is a mean rise in IOP by $2.27 \mathrm{mmh}$ which is statistically significant (Table 3).

Table 1: Distribution of Age group among study subjects

\begin{tabular}{|l|c|c|c|}
\hline \multicolumn{2}{|c|}{} & Frequency & $\%$ \\
\hline \multirow{3}{*}{ Age Group } & Less than 60 Years & 45 & $76.3 \%$ \\
\cline { 2 - 4 } & Between 61 to 65 Years & 10 & $16.9 \%$ \\
\cline { 2 - 4 } & More than 65 Years & 4 & $6.8 \%$ \\
\hline
\end{tabular}

Graph 1: Graph wise distribution of Age group

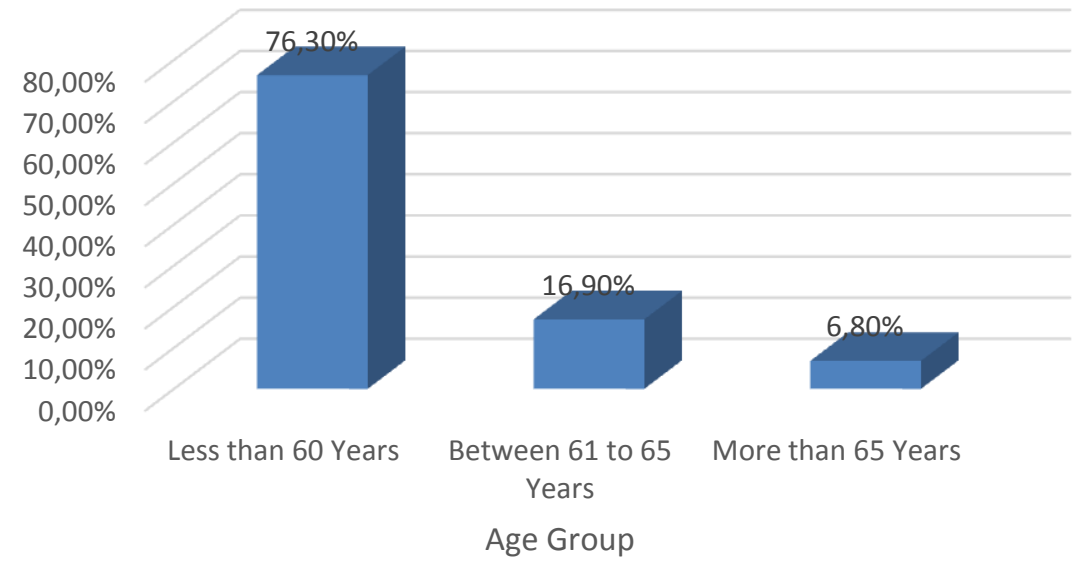


Table 2: Mean Distribution of Intra Ocular Pressure among study subjects Before Surgery and @ 6 Weeks

\begin{tabular}{|l|c|c|}
\hline & Mean & Standard Deviation \\
\hline Pre OP IOP & 17.05 & 1.36 \\
\hline 6 Weeks IOP & 19.32 & 1.69 \\
\hline
\end{tabular}

Graph 2: Line Diagram of Mean IOP among study subjects Before Surgery and @ 6 Weeks

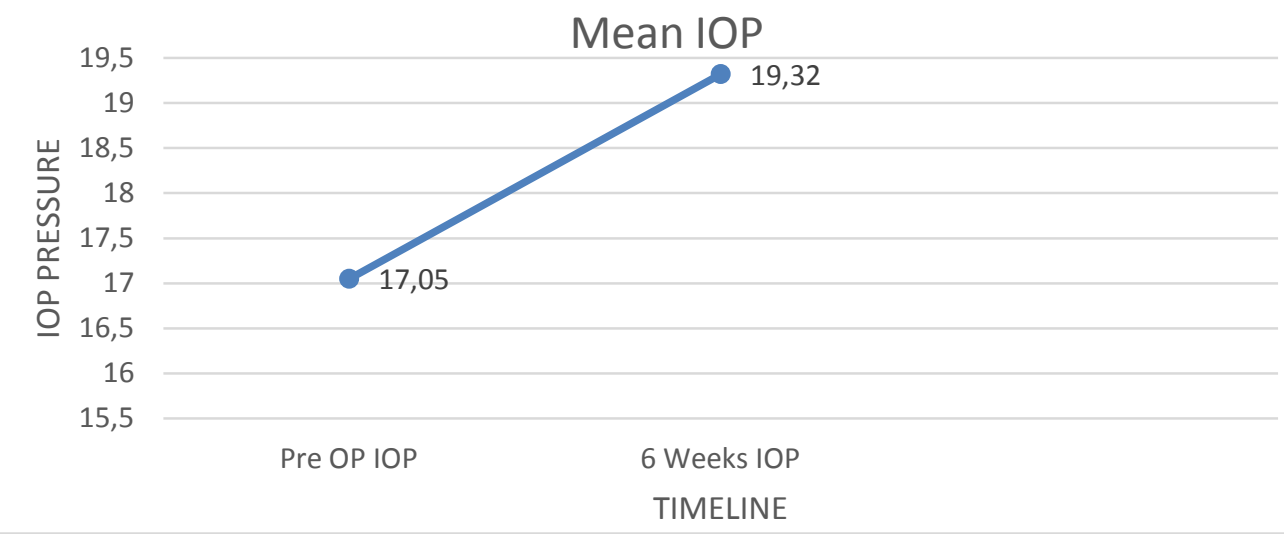

Table 3: Comparison of Mean IOP at Pre Operative and @ 6 Weeks

\begin{tabular}{|l|c|c|c|c|c|}
\hline & \multirow{2}{*}{$\begin{array}{c}\text { Mean } \\
\text { Difference }\end{array}$} & \multirow{2}{*}{$\begin{array}{c}\text { Standard } \\
\text { Error }\end{array}$} & \multicolumn{2}{|c|}{$\begin{array}{c}\text { 95\% Confidence Interval of the } \\
\text { Difference }\end{array}$} & \multirow{2}{*}{$\begin{array}{c}\text { P Value } \\
\text { (Paired t test) }\end{array}$} \\
\cline { 4 - 5 } & & Lower Limit & Upper Limit & (Paim \\
$\begin{array}{l}\text { Pre OP IOP } \\
\text { 6 Weeks IOP }\end{array}$ & -2.27119 & .14061 & -2.55266 & -1.98972 & $<0.001$ \\
\hline
\end{tabular}

\section{Discussion}

In the early 1980s, an attempt was made at providing less surgically induced astigmatism and better wound healing following cataract surgery by the introduction of the scleral tunnel incision. Following this, Blumenthal developed modern small incision cataract surgery which is widely practiced in developing countries due to its cost effectiveness, shorter learning curve and early rehabilitation. $^{2,3}$ Many studies have been conducted to report the effect of conventional cataract surgery on the intraocular pressure of the eye but there are very few studies with respect to small incision cataract surgery. Following cataract surgery, patients are started on steroid eye drops to control the postoperative pain and inflammation. It's a known fact that there is a transient rise of IOP in the immediate postoperative period but the effect of the long term use of corticosteroids on the changes in the IOP is a less studied subject. Hence, it's the need of the hour to study the effect of long term use of corticosteroid on the IOP changes and extent in the postoperative cataract patients as it can lead to deleterious effects on steroid responders and to prognosticate in patients of glaucoma and ocular hypertension. ${ }^{4,5}$

The present study is a prospective analysis of variation and extent of the variation in IOP after steroid usage for 6 weeks following manual small incision cataract surgery.

In this study we included 59 subjects and majority of the patients were below 60 years of age (76.3\%) and between 61-65 years of age (16.9\%) which corroborates with study done by PD Sharma and MR Madhavi. ${ }^{4}$

In our study, consisting of 59 subjects, the mean preoperative IOP was $17.05 \mathrm{mmhg}$, which postoperatively increased by $2.27 \mathrm{mmhg}$ ( $\mathrm{p}<0.001$, statistically significant) at 6 weeks.

In a study conducted by Sahasrabudhe Vivek M, Kamble Nikhil R on 56 patients to study the effect of topical Difluprednate on IOP in patients operated by SICS concluded that there was a rise 
in IOP in only $2(3.57 \%)$ patients. In these patients the postoperative IOP was measured at 6 weeks by Goldmann Applanation tonometry and was found that strict following of dosing is necessary and there is a low risk of IOP rise if the dosing is followed, ${ }^{6}$ which very much corroborates with our present study.

A hospital based cross sectional observational study was conducted by Sanaa A et al to determine the distribution of IOP and its association with age. The study concluded that there is no statistically significant difference in IOP in relation to the age $(\mathrm{p} \text { value }=0.751)^{7}$ which corroborates with our present study showing no statistical significance in the pre operative IOP between the age group of less than 60 years, 61-65 years and more than 65 years $(\mathrm{p}$ value $=0.450)$. Similarly, there was no statistical significance in the IOP at 6 weeks ( $p$ value $=0.921$ ). In our study, there was a rise in IOP of less than 10mmhg at 6 weeks post operation.

\section{Summary}

We analyzed 59 patients who were operated by Manual Small Incision Cataract extraction and started on Dexamethasone in tapering doses over 6 weeks. The Pre-operative IOP was compared with the post op IOP at 6 weeks of Dexamethasone usage.

Out of all the 59 patients operated for cataract extraction, majority of the patients were in the age group of less than 60 years $(76.3 \% 0)$ followed by 61-65 years (16.9\%) and the least number of patients were in the age group of more than 65 years $(6.8 \%)$

The mean pre-operative IOP among all the study subjects was found to be $17.05 \mathrm{mmh}$ which rose to $19.32 \mathrm{mmhg}$ after the usage of Dexamethasone for 6 weeks in the postoperative period.

A statistical significance was found between the pre operative IOP in comparison with the IOP at 6 weeks following Dexamethasone usage. There was no statistical significance between the association of IOP with the age of undergoing cataract extraction.

\section{Conclusion}

Dexamethsone is a safe and potent corticosteroid which effectively reduces the post operative inflammation and pain. Dexamethasone should be cautiously used in patients with glaucoma. All the post operative patients receiving topical ocular Dexamethasone should be followed regularly and be cautioned about the rise in IOP causing pain and drop in the vision. The patient compliance with respect to the frequency of instillation of Dexamethasone is most important to obtain good outcome. Any patient presenting with the acute rise in IOP should be withdrawn of Dexamethasone, termed as Dexamethasone responder and treated with adjuvant medications. Future use of Dexamethasone in such patients warrants utmost caution. We conclude that Dexamethasone is a safe drug which carries very low risk of IOP rise if the schedule is followed.

\section{Acknowledgement: Nil \\ Financial Support: Nil}

\section{References}

1. Pascolini D, Mariotti SP. Global estimates of visual impairment: 2010. $\mathrm{Br} \mathrm{J}$ Ophthalmol. 2012 May;96(5):614-8. doi: 10.1136/bjophthalmol-2011-300539. Epub 2011 Dec 1. PMID: 22133988.

2. Blumenthal M. Manual ECCE, the present state of the art. Klin Monatsbl Augenheilkd 1994; 205(5): 266-270.

3. Blumenthal M, Glovinsky Y. Surgical consequences in coexisting cataract and glaucoma. Current Opinion Ophthalmology 1995; 6(2): 15-18.

4. Sharma PD, Madhavi MR. A comparative study of postoperative intraocular pressure changes in small incision vs conventional extracapsular cataract surgery. Eye 2010 Apr;24(4):608-12.

5. Gogate PM, Deshpande M, Wormald RP, Deshpande R, Kulkarni SR. Extracapsular cataract surgery compared with manual small incision cataract surgery in 
community eye care setting in western India: a randomised controlled trial. $\mathrm{Br} \mathrm{J}$ Ophthalmol. 2003:87(6):667-72.

6. Sahasrabudhe Vivek, M, Kamble Nikhil, R. Study of effect of topical $005 \%$ Difluprednate on Intra Ocular Pressure in patients operated by Small Incision Cataract Surgery. Saudi J Med Pharm Sci. 2016;2(3): 56-58.

7. Yassin SA, Al-Tamimi ER. Age, gender and refractive error association with intraocular pressure in healthy Saudi participants: A cross-sectional study. Saudi J Ophthalmol. 2016 Jan-Mar;30(1):44-8. doi: 10.1016/j.sjopt.2015.11.007. Epub 2015 Nov 23. PMID: 26949358; PMCID: PMC4759511. 